\title{
Magic-Angle Hopping as an Alternative to Magic-Angle Spinning for Solid State NMR*
}

\author{
Nikolaus M. Szeverenyi, Ad BaX, $\dagger$ AND Gary E. MaCiel $\ddagger$ \\ Department of Chemistry, Colorado State University, Fort Collins, Colorado 80523
}

Received May 10, 1984; revised August 20, 1984

\begin{abstract}
A new variation is presented on the recently reported two-dimensional magic-angle hopping technique. In this new experiment, the frequency domain that provides chemical-shift anisotropy information has been "sacrificed" in favor of improved sensitivity in the isotropic chemical-shift spectrum. This is accomplished by repeatedly refocusing the initial magnetization in the $t_{2}$ (data acquisition) domain and measuring the resulting string of echoes. Although the sensitivity is still inferior to that of a standard magic-angle spinning (MAS) experiment, it far surpasses that achieved in the original 2$\mathrm{D}$ approach, and may render the hopping approach an attractive alternative to MAS in some kinds of applications. 1985 Academic Press, Inc.
\end{abstract}

\section{INTRODUCTION}

In a previous communication ( 1 ) we reported a technique that averages the chemical-shift anisotropy (CSA) for powdered, solid samples. This new technique, which we called "magic-angle hopping," relies on rapid, discrete sample rotations in conjunction with appropriately timed radiofrequency pulses. The advantage of this technique is that it is carried out as a 2-D experiment and yields both isotropic chemical shifts and the corresponding CSA powder patterns. A limitation is, however, that the signal finally obtained is one that decays very rapidly (the sample is stationary during the detection period), and the sensitivity of the technique is correspondingly low.

For many applications the CSA powder patterns are of only secondary interest, and one would be willing to sacrifice this information for better sensitivity in the CSA-averaged spectrum. Accordingly, we have developed a technique based on the experiment referred to above, but utilizing a train of echo pulses applied during the detection period. This modification samples only the initial value of the magnetization during the detection $\left(t_{2}\right)$ period; however, the technique still provides isotropic chemical-shift information, which is generated during the evolution $\left(t_{1}\right)$ period, but with sensitivity approaching that of the magic-angle spinning (MAS) experiment. This technique should find utility for experiments in which large chemical-shift anisotropies are encountered, where very rapid sample rotation would otherwise be

* Presented in part at the 24th ENC, Asilomar, Calif,, April 1983.

$\dagger$ Present address: National Institutes of Health, Bethesda, Md. 20205.

¥ To whom correspondence should be addressed. 
necessary with the magic-angle spinning experiment. Such cases include ${ }^{13} \mathrm{C}$ experiments at high magnetic fields and experiments with nuclides with extremely large CSAs (e.g., heavy-metal nuclides or ${ }^{31} \mathrm{P}$ ).

Since sample "hops" are relatively slow, a simple mechanical system can be employed to rotate the sample. This system allows larger sample volumes and lends itself to temperature regulation more readily than does the MAS system. Furthermore, since the sample is stationary for most of the experiment other techniques may be employed to generate magnetization, techniques which normally do not work well under magic-angle spinning conditions, e.g., ADRF or cross polarization between weakly coupled nuclei.

\section{EXPERIMENTAL}

Spectra were obtained at a ${ }^{13} \mathrm{C}$ frequency of $25.27 \mathrm{MHz}$ with a homemade spectrometer system having a 3.7 $\mathrm{T}$ Nalorac superconducting magnet operated at $2.3 \mathrm{~T}$. A Nicolet 1180 computer and $293 \mathrm{~B}$ pulse programmer were used to control the experiment. The phase cycling information for the $\mathrm{ff}$ pulses needed to reconstruct the isotropic chemical shifts was stored in the main memory of a "black box" from AdNic Products (Chemagnetic, Inc.) and operated in conjunction with the pulse programmer.

The probe is of a single solenoid-coil design, doubly tuned for ${ }^{13} \mathrm{C}$ and ${ }^{1} \mathrm{H}$. The coil dimensions are 6 turns of $2.0 \mathrm{~mm}$ diameter copper wire, with an inside diameter of $16.5 \mathrm{~mm}$. $\left({ }^{13} \mathrm{C} \pi\right.$ pulses were $\left.14 \mu \mathrm{s}\right)$. The sample was contained in a hollow KelF cylinder with an internal volume of $1.9 \mathrm{~cm}^{3}$ and was attached to a gear mechanism that generated rotation of the sample about an axis inclined $54.7^{\circ}$ to the external magnetic field. The sample is rotated inside the if coil under computer control via a stepper motor, as depicted in Fig. 1. The stepper motor (Airpax K82701-P2) has a $7.5^{\circ}$ step size with a resolution of $0.5^{\circ}$. Each sample rotation was $120^{\circ}$, requiring 16 steps of the motor, furnished by a simple TTL counter and motor drive circuitry. A $120^{\circ}$ sample rotation required ca. $150 \mathrm{~ms}$.

The basic experiment used in this study is shown schematically in Fig. 2. Prior to the detection period, this experiment differs from what was reported earlier (1) in the addition of a third hop between the last $t_{1} / 3$ period and detection; this additional hop was added only for technical convenience and in no way alters the strategy (since the sample is static during detection and one orientation of the powder sample is as good as any other during this period). In these experiments the sweep width for quadrature data acquisition was $\pm 2000 \mathrm{~Hz}$, corresponding to digitization of a sample point every $250 \mu \mathrm{s}$. The ${ }^{13} \mathrm{C}$ refocusing pulses ( $\pi$ pulses) were applied midtime between these data acquisition times so as to produce an echo exactly at each point in time when the data acquisition occurred. The receiver was gated off during the ${ }^{13} \mathrm{C}$ pulse and for a time $50 \mu \mathrm{s}$ thereafter. In this manner a train of 100 echoes was collected for each of the eight experiments carried out for every increment of the evolution time, $t_{1}$. Eight is the minimum number of experiments (echo trains) to be carried out for each value of $t_{1}$ in order to cycle through the desired scheme of pulse phases, as presented in Table 1, and described below. The spectrum of $p$-dimethoxybenzene presented in Fig. 3 was carried out 


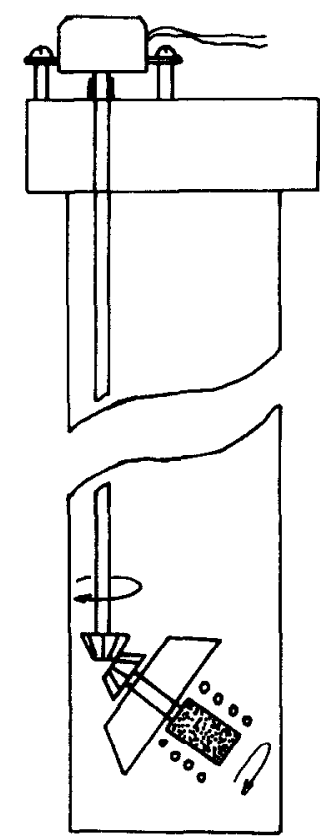

FIG. 1. A schematic diagram of the magic-angle hopping probe. The stepper motor turns a long drive shaft and, via a set of bevel gears, rotates the sample inside the receiver coil. The axis of the sample rotation is adjusted to make an angle of $54.7^{\circ}$ relative to the static magnetic field.

with this minimum number of echo trains for each $t_{1}$ and was obtained in a total experiment time of approximately $20 \mathrm{~min}$.

\section{RESULTS AND DISCUSSION}

Chemical-shift anisotropy is a property that often gives rise to complications in the NMR spectra of solid samples. To get high-resolution chemical-shift information from solids, it is usually necessary to average this interaction by some external means. In the past this has been carried out by very rapid mechanical rotation of

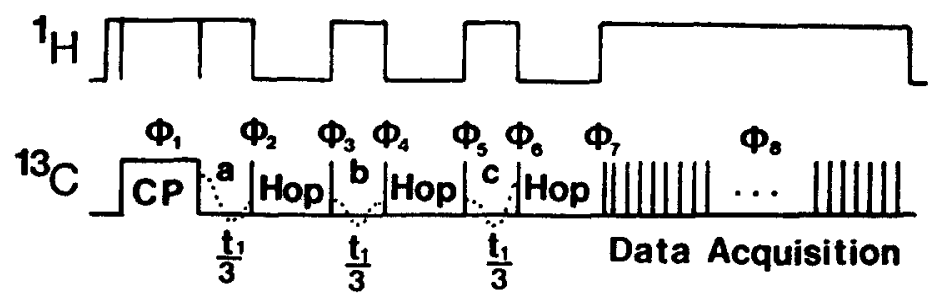

FIG. 2. A schematic diagram of the pulse sequence for magic-angle hopping with echoes during the detection period. The phases of the $\pi / 2$ pulses are given by $\phi_{2}$ through $\phi_{7}$ and the phase of the $\pi$ echo pulses is given by $\phi_{8}$ and are specified in Table 1. "Hop" describes the interval when the sample is actually rotating, and the designations " $a$," " $b$," "c" refer to the three sample orientations related by a $120^{\circ}$ rotation about the magic-angle axis. 
TABLE 1

Radiofrequency Phases for Pulses in the Sequence of Fig. 2

\begin{tabular}{|c|c|c|c|c|c|c|c|c|c|c|c|c|}
\hline \multirow{2}{*}{$\begin{array}{l}\text { Expt } \\
\text { No. }{ }^{a}\end{array}$} & \multicolumn{8}{|c|}{ rf phase ${ }^{b}$} & \multirow[b]{2}{*}{$\mathrm{Acq}^{c}$} & \multicolumn{3}{|c|}{$\begin{array}{l}\text { Pertinent trigonometric } \\
\text { Factor }^{d}\end{array}$} \\
\hline & $\phi_{1}$ & $\phi_{2}$ & $\phi_{3}$ & $\phi_{4}$ & $\phi_{3}$ & $\phi_{0}$ & $\phi_{7}$ & $\phi_{8}$ & & "a" & "b" & "c" \\
\hline 1 & $x$ & $y$ & $-y$ & $y$ & $-y$ & $y$ & $-y$ & $x$ & + & $\cos$ & $\cos$ & $\cos$ \\
\hline 2 & $x$ & $-x$ & $-y$ & $y$ & $x$ & $-x$ & $x$ & $y$ & + & $i \sin$ & $\cos$ & $\cos$ \\
\hline 3 & $x$ & $y$ & $-y$ & $x$ & $x$ & $-x$ & $x$ & $y$ & - & $-i \cos$ & $\sin$ & $\cos$ \\
\hline 4 & $x$ & $-x$ & $-y$ & $-x$ & $-y$ & $y$ & $-y$ & $x$ & - & $\sin$ & $\sin$ & $\cos$ \\
\hline 5 & $x$ & $y$ & $-y$ & $y$ & $-y$ & $-x$ & $x$ & $y$ & + & $i \cos$ & $\cos$ & $\sin$ \\
\hline 6 & $x$ & $-x$ & $-y$ & $y$ & $x$ & $-y$ & $y$ & $-x$ & + & $-\sin$ & $\cos$ & $\sin$ \\
\hline 7 & $x$ & $y$ & $-y$ & $x$ & $x$ & $-y$ & $y$ & $-x$ & - & $\cos$ & $\sin$ & $\sin$ \\
\hline 8 & $x$ & $-x$ & $-y$ & $-x$ & $-y$ & $-x$ & $x$ & $y$ & - & $i \sin$ & $\sin$ & $\sin$ \\
\hline
\end{tabular}

${ }^{a}$ Expt No. refers to the specific pulse sequence in the collection of eight required for a particular value of $t_{1}$.

${ }^{b}$ Radiofrequency pulse phases $\phi_{1}-\phi_{8}$ shown in Fig. 2. Pulses 2 through 7 are $90^{\circ}$ pulses. Pulses of the type 8 are $180^{\circ}$ pulses.

${ }^{c}$ Sign of the acquisition into memory.

${ }^{d}$ The last three columns provide the trigonometric function for the pertinent magnetization component during each of the indicated orientations, " $a$ ", " $b$ ", and "c".

the sample about the so-called magic-angle axis (2), resulting in each molecule's experiencing a continuous series of orientations with respect to the external magnetic field. Although dephasing of transverse magnetization occurs during this rotation because of chemical-shift anisotropy, at the end of a complete rotor period the spins refocus in a rotational echo (3); only the contribution from the isotropic chemical shift is not refocused. In principle one could collect signals at only these echo tops, thereby eliminating the effects of CSA dephasing; but, because of constraints imposed by the sampling theorem, this would produce a sweep width too narrow for general use in ${ }^{13} \mathrm{C}$ NMR spectroscopy. Sampling between echo tops leads to the problem of spinning sidebands.

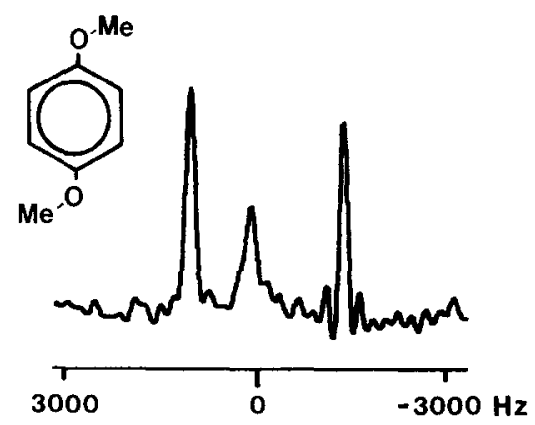

FIG, 3. ${ }^{13} \mathrm{C}$ NMR spectrum of 1,4-dimethoxybenzene obtained from the hopping experiment with the echo sequence applied during data acquisition. 
The hopping experiment in this context can be thought of as a technique which allows one to generate by addition of a suitable combination of four spin responses (1) a net spin response that is analogous to a rotational echo, but an "echo" that can be produced at an arbitrary time, $t_{1}$. By doing this as a two-dimensional type of experiment, one samples this "composite rotational echo" at incremented values of the evolution-time parameter, $t_{1}$, the modulation of this "echo" thereby carrying the information on isotropic chemical shifts. With a series of experiments of increasing $t_{1}$ values, what amounts to an isotropic-shift interferogram is generated. Each point in this interferogram can be thought of as a measure of the amplitude of a "composite rotational echo" collected at an apparent "rotor period" of $3\left(t_{1} / 3\right)$.

In the previous communication $(I)$ this "composite rotational echo" was produced in a sample that was stationary during the detection period and gave rise to a very rapidly decaying signal, the initial amplitude of which reflected the "composite rotational echo" amplitude discussed above. The modification we suggest here is a technique similar to that used by Waugh and Wang (4), and consists of a series of ${ }^{13} \mathrm{C} \pi$ pulses applied during the detection period. These pulses have the property of refocusing a component of ${ }^{13} \mathrm{C}$ magnetization in a series of echoes (not to be confused with the rotational echos discussed above). The generation of these echoes is depicted in Fig. 4, which is concerned with the detection period. The vector diagrams of that figure show how the "composite rotational echo" formed by the correct combination of spin responses from four sequences (actually eight sequences, as seen below) behaves during the detection period. At the beginning of the detection period $\left(t_{2}=0\right)$ the phase of this composite (from four experiments) magnetization vector $\left(M_{\mathrm{c}}\right)$ is zero, because the $t_{1}$-modulated magnetization has been rotated down from its "storage" along the $z$ axis. This initial phase is the same for all crystallites in the system. However, as the sample is stationary during the detection period, the

a)

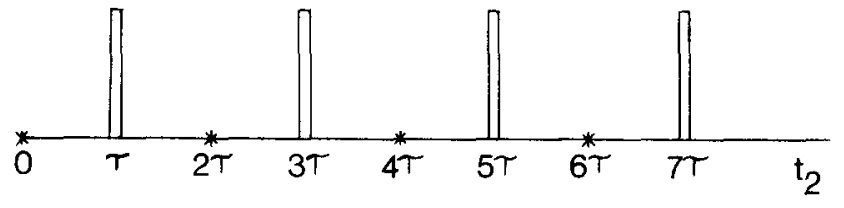

b)
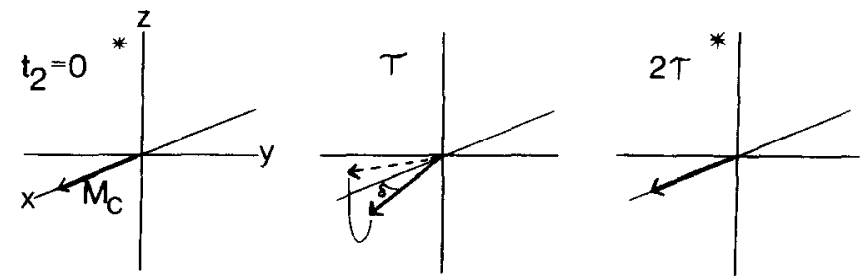

FIG. 4. Echo pulse sequence (a) and response of the composite rotational echo in the $x y$ plane during the detection period (b). Each pulse is a $\pi$ pulse, with phase indicated as $\phi_{8}$ in Table $1 . \delta$ is the angle accumulated during the period $\tau$ by a spin isochromat with a specific crystallite orientation. The dashed line in (b) corresponds to the position of the magnetization vector at the end of a $\pi$ pulse. Asterisks show the times of data acquisition. 
continued evolution of the magnetization depends upon crystallite orientation. For some arbitrary orientation the composite magnetization vector changes phase by the amount $\delta$ during the time between $t_{2}=0$ (where the first data point is taken) and the point at which the first $\pi$ pulse occurs. It is readily seen that the $x$ component of the composite magnetization vector is $M_{\mathrm{c}}$ at each time when a data point is taken, neglecting relaxation, the chemical-shift evolution being refocused at each data acquisition time in a Carr-Purcell-Meiboom-Gill echo. The same type of behavior will be executed for any crystallite orientation. These echoes can be made to persist for a time determined by the intrinsic homogeneous $T_{2}$ values of individual spin isochromats, which is much longer than the inhomogeneous $T_{2}$ obtained for the CSA-broadened line of the stationary sample. Echo trains can be made to last for tens of milliseconds, as shown in Fig. 5.

Instead of detecting the rapidly decaying signal of a stationary powder pattern, as done in our previous communication (with a $t_{2}$ dependence giving rise to the CSA pattern in the corresponding frequency domain), in the present modification the echo tops are detected, which can be thought of as repeatedly sampling the first point of the static-powder-pattern FID. The noise in these echo tops is uncorrelated, which leads to a substantial increase in signal-to-noise ratio for the measurement of the initial magnetization intensity (which is modulated in $t_{1}$ ). As the echo top



FIG. 5. Echo trains during the detection period for 1,4-dimethoxybenzene. In the Carr-PurcellMeiboom-Gill (CPMG) sequence the rf $\pi$ pulses are applied with a phase that makes $H_{1}$ parallel to the initial magnetization. In the Carr-Purcell (CP) sequence the phase of the $\pi$ pulses is $90^{\circ}$ relative to the phase of the initial magnetization, which accounts for the alternation of sign in the echoes. In the CPMG sequence pulse imperfections cancel after every second pulse and echo trains persist for a long time. In the CP sequence pulse errors in the $\pi$ pulses accumulated, causing fast decay of magnetization. 
intensity decreases due to $T_{2}$ and pulse imperfections, the signal content decreases relative to the noise. The calculation of the magnetization intensity should weight the later points less strongly than the initial ones. One convenient scheme to process the data is to treat the echo train as a FID and apply an exponential filtering (weighting) function, and then Fourier transform the resulting data. This yields a narrow peak at $0 \mathrm{~Hz}$. Transposing the point corresponding to the top of this peak for the various " $0 \mathrm{~Hz}$ spectra" with incremented $t_{1}$ values leads to the construction of the FID containing isotropic chemical shifts. When transformed, this gives highresolution spectra of the type shown in Fig. 3.

Since sample "hops" are relatively slow compared to the evolution of transverse magnetization in the three static orientations, in order to avoid dephasing during hops, a technique must be applied to store the transverse spin information after each $t_{1} / 3$ evolution time. The technique used here, as before (1), is the application of a $\pi / 2$ pulse, storing either the $x$ or the $y$ component of the transverse magnetization along $z$. (Whether it is the $x$ or $y$ component depends on the phase of the $\pi / 2$ pulse.) After the hop the magnetization must be returned from the $z$ axis to the transverse plane (via another $\pi / 2$ pulse) to undergo further evolution in the new sample orientation. Since only one component of the $x y$ magnetization can be stored (and preserved) in this manner during the first $t_{1} / 3$ period, two separate experiments must be carried out to record the entire spin information in the $x y$ plane. After evolution in the second orientation the transverse spin information must again be stored as before, requiring an additional two experiments to retain the full spin information. It is a combination of magnetization vectors resulting after the final $t_{1} / 3$ evolution period, in the third orientation, that forms the "composite rotational echo." It is the amplitude of this "echo," as modulated in $t_{1}$, that contains the isotropic shift spectrum; and one tries to measure this amplitude by applying the $\pi$-pulse string to form the echo train described above. At this point it would seem that only four experiments are required to reconstruct the isotropicshift FID, as was the case in our earlier communication (I).

According to the scheme outlined in Figs. 2 and 4, the evolution of only one component of the transverse magnetization during the third $t_{1} / 3$ period of the four experiments described above is monitored during the echo train in the $t_{2}$ period. To record the evolution of the second component of $x y$ magnetization during the third $t_{1} / 3$ period, i.e., to specify completely the "composite rotational echo," one must carry out another set of four experiments, giving rise to a total of eight experiments required. The combination of pulse phases employed in these eight experiments is summarized in Table 1.

Figure 5 summarizes results obtained from various approaches to constructing echo trains, and provides a comparison of results obtained with the Carr-PurcellMeiboom-Gill (CPMG) sequence (5) and those of the Carr-Purcell (CP) sequence (6). The spectra were obtained for 1,4-dimethoxybenzene, and give results as a function of pulse spacing. The only difference between the sequences for which results are shown in Fig. 5 is the phase of the $\pi$ pulses relative to the direction of the original magnetization vector at $t_{2}=0$. It is seen that much better refocusing is obtained if the direction of $H_{1}$ for the $\pi$ pulses is colinear with the magnetization vector at $t_{2}=0$ (CPMG); i.e., under these conditions the echoes persist much 
longer. Taking the situation of very closely spaced $\pi$ pulses to the limit of continuously joined pulses, this colinear case approaches the spin-lock approach. The use of composite $\pi$ pulses (7) to correct for pulse imperfections did not seem to improve the persistence of the echo train very much.

\section{CONCLUSION}

Although somewhat more demanding in terms of pulse programmer capabilities and subsequent data work up, this experiment provides a means of obtaining highresolution NMR data on powdered solids that might not be available by the conventional CP/MAS technique. The kind of situation in which the modified magic-angle hopping technique might be advantageous could arise either because of MAS interference with the cross-polarization technique or because of the difficulty of making MAS mechanically compatible with the desired chemical and/or physical conditions under which one wishes to maintain the sample. Sensitivity is not as high as for magic-angle spinning, but in many cases this is not a crucial factor.

One final issue, that of resolution, has not been addressed above. In the present work resolution is still not as good as with the MAS experiment. The resolution obtained in the hopping experiment is limited by the maximum length of the $t_{1}$ interval employed. This means that an experiment to obtain higher resolution requires a longer total elapsed time, especially if one wishes to maintain the same spectral width. The tolerances of the mechanism used to hop the sample can also be a factor influencing resolution. Not only does the angle of the hopping axis have to be adjusted accurately to the magic angle, but the size of each hop should be exactly $120^{\circ}$. (This may still be a significant problem in our first, rather primitive hopping probe; a more sophisticated device with better mechanical tolerances is now being tested.) Finally, the effects of carbon-carbon dipolar couplings may not be negligible in this experiment, but this contribution becomes relatively less important as the strength of the static magnetic field is increased. Experiments are currently underway to determine how to optimize this technique and what its limitations are.

\section{ACKNOWLEDGMENTS}

The authors gratefully acknowledge the partial support of this work by grants from the U.S. Geological Survey and from the National Science Foundation (CHE-8306518).

\section{REFERENCES}

I. A. BaX, N. M. Szeverenyi, AND G. E. MaCiel, J. Magn. Reson. 52, 147 (1983).

2. J. Schaefer and E. O. Stejskal, J. Am. Chem. Soc. 95, 1031 (1976).

3. M. MaricQ and J. S. Waugh, J. Chem. Phys. 70, 330 (1979).

4. J. S. Waugh ANd C. H. Wang, Phys. Rev. 162, 209 (1967).

5. S. MEIBOOM AND D. GiLl, Rev. Sci. Instrum. 29, 688 (1958).

6. H. Y. Carr and E. M. Purcell, Phys. Rev. 94, 630 (1954).

7. M. H. LeVitt AND R. Freeman, J. Magn. Reson. 43, 65 (1981). 